\title{
Caracterización del mercado microempresarial ecuatoriano: el caso de la industria de la confección textil
}

\author{
Wilson Abad León \\ Universidad Andina Simón Bolívar, Sede Ecuador \\ wilson.abad.leon@gmail.com

\section{Wilson Araque} \\ Universidad Andina Simón Bolivar, Sede Ecuador \\ wilson.araque@uasb.edu.ec
}

Fecha de presentación: 9 de agosto de 2015 • Fecha de aceptación: 11 de octubre de 2016

Artículo de investigación 


\section{Resumen}

Con el propósito de conocer mejor a los clientes y desarrollar acciones que beneficien la relación entre las entidades financieras operadoras de microcrédito y los clientes, se plantea alternativas metodológicas de análisis de las variables de comportamiento que puedan influir en las decisiones financieras de los microempresarios. Se utilizó modelos psicoeconómicos validados en mercados de consumidores finales y adaptados a las condiciones de los microempresarios para evaluarlos y caracterizarlos en: actitud hacia el endeudamiento, estilo de vida y actitud hacia las entidades financieras, y hábitos y conductas de consumo.

Palabras clave: microcrédito, microempresa, caracterización, método multivariable, segmentación.

JEL: C3 Métodos econométricos; L67 Otros bienes de consumo perecederos: vestido, textiles.

\section{Abstract}

With the purpose of getting to know the clients better and develop actions that benefit the relationship between the microcredit operating financial entities and the clients, methodological alternatives of analysis of the behavioral variables that may influence the financial decisions of the micro-entrepreneurs are considered. Psycho-economic models validated in final consumer markets and adapted to the conditions of micro-entrepreneurs were used to evaluate and characterize them in: attitude towards indebtedness, lifestyle and attitude towards financial entities, and consumption habits and behaviors.

Keywords: microcredit, microenterprise, characterization, multivariable method, segmentation. JEL: C3 Econometric methods; L67 Other perishable consumer goods: clothing, textiles.

\section{Resumo}

Com o propósito de conhecer melhor os clientes e desenvolver ações que beneficiem sua relação com as entidades financeiras operadoras de microcrédito, projetam-se alternativas metodológicas de análise das variáveis de comportamento que possam influir sobre as decisões financeiras dos microempresários.

Os modelos psicoeconômicos que servem de base para esta pesquisa foram validados por seus autores em mercados de consumidores finais e adaptados às condições dos microempresários para avaliá-los e caracterizá-los quanto a: sua atitude frente ao endividamento, seu estilo de vida e sua atitude frente às entidades financeiras e seus hábitos e condutas de consumo.

Palavras-chave: Microcrédito, microempresa, caracterização, método multivariável, segmentação. JEL: C3 Métodos econométricos; L67 Outros bens de consumo não duráveis: roupas, têxteis. 


\title{
Introducción
}

\begin{abstract}
A partir de 1995, el interés por el desarrollo de la microempresa en Ecuador se hace evidente con la aparición de nuevas entidades fi1 nancieras y la incursión de bancos tradicionales (Berger, Goldmark y Miller-Sanabria 2007); crecimiento que, para uno de los nuevos bancos operadores de microcrédito, en 2005 significó alcanzar la cifra de 148.712 clientes, con una cartera de 183 millones de dólares y un préstamo promedio de US \$ 909. En el período 2002-2009 (figura 1) la evolución de las entidades microfinancieras pasaron de 16 a 67, lo que significó un crecimiento del
\end{abstract}

Figura 1

\section{Evolución de las entidades con cartera de microfinanzas}

\begin{tabular}{|c|c|c|c|c|c|c|c|c|}
\hline & & & úmerc & entid & & & & \\
\hline 35 & & & & & & & & \\
\hline 30 & & & & & & & & \\
\hline 20 & & & & & & & & \\
\hline 10 & & & & & & & & \\
\hline & & 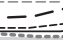 & & & & & 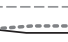 & \\
\hline & 2002 & 2003 & 2004 & 2005 & 2006 & 2007 & 2008 & 2009 \\
\hline-- Bancos & 2 & 3 & 7 & 13 & 16 & 16 & 19 & 19 \\
\hline Cooperativas & 11 & 22 & 31 & 36 & 36 & 36 & 36 & 37 \\
\hline Mutualistas & 1 & 1 & 1 & 2 & 2 & 2 & 1 & 1 \\
\hline ------ Sociedades financieras & 1 & 1 & 3 & 6 & 6 & 6 & 7 & 7 \\
\hline ...... Banca pública & 1 & 0 & 0 & 1 & 1 & 2 & 2 & 2 \\
\hline
\end{tabular}

Fuente: Superintendencia de Bancos y Seguros.

Elaboración: Observatorio de la PyME, UASB-E. 
$319 \%$; el mayor crecimiento se observa en las cooperativas de ahorro y crédito controladas, pasando de 11 a 36 en 2005; mientras que la banca pública incrementó tres entidades más para 2009.

Para 2009, la cartera bruta de microcrédito del sistema financiero alcanzó la suma de US \$ 1.618 millones, lo que significó un incremento del 145\% respecto a 2005. El comportamiento de la cartera de microcrédito se detalla a continuación.

Figura 2

Total cartera bruta de microcrédito

\begin{tabular}{|c|c|c|c|c|c|c|c|c|}
\hline & & & Miles c & e dólares & & & & \\
\hline 800.000 & & & & & & & & \\
\hline 700.000 & & & & & & & & \\
\hline 500.000 & & & & & & & & \\
\hline 400.000 & & & & & & & & \\
\hline $\begin{array}{l}300.000 \\
200.000\end{array}$ & & & & & & & & \\
\hline 100.000 & & & & & & & & \\
\hline & 2002 & 2003 & 2004 & 2005 & 2006 & 2007 & 2008 & 2009 \\
\hline - - Bancos & 56.663 & 99.196 & 201.980 & 353.999 & 483.053 & 608.158 & 831.464 & 842.667 \\
\hline Cooperativas & 5.533 & 50.179 & 112.542 & 284.012 & 354.134 & 443.298 & 557.855 & 601.433 \\
\hline - Mutualistas & 1.136 & 864 & 577 & 357 & 265 & 177 & 585 & 1.357 \\
\hline ----- Sociedades Financieras & 7.071 & 17.156 & 13.255 & 20.903 & 28.089 & 33.066 & 21.194 & 33.081 \\
\hline -.---- Banca Pública & 2.831 & & - & 1 & 1.665 & 28.368 & 103.159 & 139.486 \\
\hline
\end{tabular}

Fuente: Superintendencia de Bancos y Seguros.

Elaboración: Observatorio de la PyME, UASB-E.

El interés por este mercado permitió que entidades como la Agencia para el Desarrollo Internacional de los Estados Unidos de América-Proyecto SAL$\mathrm{TO}^{1}$ publique un estudio que caracterice al mismo (Magill y Meyer 2005, 104). El estudio en mención aborda el tema, por un lado, mediante variables de tipo demográfico de los microempresarios - género, edad, educación, estado civil, tamaño de familia y posición familiar-; y por otro, caracteriza a las microempresas mediante variables como tamaño, sector, antigüedad,

1. Strengthen Access to Microfinance and Liberalization Task Order. 
experiencia de negocios, formalidad, entre otros; en el mismo estudio, se manifiesta que "las instituciones de microfinanzas ecuatorianas necesitan estar cada vez más conscientes de las actitudes del cliente, desarrollar las respuestas eficaces para capitalizar en los aspectos positivos sus relaciones con el cliente y mitigar el impacto negativo del descontento del cliente" (Magill y Meyer 2005, 104), razón por la cual se plantea la caracterización del mercado microfinanciero en términos de variables de comportamiento y mediante el uso de técnicas de análisis multivariable.

Con el propósito de corroborar la afirmación de Magill y Meyer, y definir las variables de estudio de la investigación, se realizaron entrevistas a ejecutivos de entidades financieras públicas y privadas operadoras de microcrédito. En dichas entrevistas, las preguntas se orientaron hacia las políticas de crédito, el conocimiento que tienen de los clientes microempresarios en términos de variables financieras, demográficas, estilos de vida y de comportamiento.

Un aspecto muy importante, y que constituye un denominador común en la información cualitativa actualizada en este estudio, es el rol de la fuerza comercial de las entidades de microcrédito para llegar al cliente, formada por asesores de crédito; por ejemplo, en una de las frases fuerza identificadas se manifiesta: "tenemos un esquema relacional con el cliente, el cliente no va a la organización sino nosotros vamos al cliente, tratamos de mantener una relación cercana". Sin embargo, el conocimiento cualitativo y cuantitativo que se tiene de los clientes, en términos generales, se enfoca al manejo y administración del negocio, así como variables históricas de compras y ventas.

Otro aspecto a resaltar es que, a través de la información cualitativa analizada, se logra evidenciar el aspecto subjetivo y cualitativo que tienen los asesores de crédito respecto al comportamiento de sus clientes; uno de ellos manifestaba, por ejemplo: "en la parte cualitativa vemos aspectos de personalidad del microempresario, características organizativas y características de relaciones con el entorno". También destacan la importancia y la necesidad de contar con información cuantitativa desde el punto de vista del comportamiento, información que habrá de ir recabando y armando, si se quiere, en un sistema que permita conocer y caracterizar a los clientes.

La dificultad de tratar de llegar a una definición de microempresa universalmente aceptada ha hecho que las diferentes organizaciones tengan diferentes necesidades y objetivos y traten de ajustar la definición a sus requi- 
sitos específicos con relación a sus segmentos de mercado especial (Magill y Meyer 2005, 4); por lo que, considerando la heterogeneidad de los diversos sectores microempresariales -los mismos que tienen sus características propias-, la investigación se orientó al sector de la confección textil.

\section{Enfoque teórico}

Para direccionar adecuadamente la investigación, se ha planteado la premisa de que el desarrollo o éxito de una microempresa probablemente dependa en gran medida del comportamiento individual o familiar de sus propietarios; por lo que se ha procedido a una revisión y análisis bibliográfico de los diferentes aportes al desarrollo de la psicología económica del consumidor.

Cuando un consumidor realiza compras -en este caso el microempresario-, se compromete con diferentes tipos de procesos de solución de problemas (McCarthy y Perreault 2001; y Shiffman y Lazar Kanuk 2005), por lo que el esfuerzo tanto mental como físico que los compradores dedican a la solución de problemas varía de manera considerable; y este depende del grado de interés por un bien o servicio y de la importancia que le asigna, denominada nivel de participación, la cual puede tener las siguientes formas: comportamiento de respuesta rutinario, solución limitada de problemas, solución ampliada de problemas y compra por impulsos.

En cualquiera de estos procesos de decisión de compra, existen posibles influencias de orden personal, psicológico y social, como se describe en la tabla 1.

Para definir el modelo psicoeconómico medible y que caracterice al mercado microempresarial ecuatoriano y particularmente al sector de la confección textil, se ha considerado el modelo psicoeconómico planteado y validado por Rodríguez Vargas (2006), el mismo que está desarrollado sobre la base del Modelo de Integración de Variables Económicas y Psicológicas sobre la Conducta del Consumidor (Van Raaij 1981) y los aportes del estudio de Denegri, Palavecinos y Ripoll (1998), que sistematizan y resumen los principales tópicos en el ámbito de la psicología económica mediante constructos ${ }^{2}$ para medir la actitud hacia el endeudamiento, hábitos y conductas de consumo.

2. Un constructo - para este caso- es entendido como una propiedad que se supone posee una persona, la cual permite explicar su conducta en determinadas ocasiones. 
Tabla 1

Decisiones del comprador

\begin{tabular}{|l|l|}
\hline Factores personales & $\begin{array}{l}\text { - Demográficos. } \\
\text { - Estilo de vida. } \\
\text { - Situacionales. }\end{array}$ \\
\hline Factores psicológicos & $\begin{array}{l}\text { - Percepción. } \\
\text { - Motivos. } \\
\text { - Aprendizaje. } \\
\text { - Actitudes. } \\
- \text { Personalidad y autoconcepto. }\end{array}$ \\
\hline Factores sociales & $\begin{array}{l}\text { - Familia. } \\
\text { - Grupos de referencia y líderes de opinión. } \\
\text { - Clases sociales. } \\
\text { - Cultura y subcultura. }\end{array}$ \\
\hline
\end{tabular}

Fuente: adaptación de Shiffman y Lazar Kanuk (2005).

Elaboración propia.

\section{El modelo de integración sobre la conducta económica de Fred van Raaij}

Este modelo general de psicología económica, citado por De Chocano (2003) y Rodríguez Vargas (2006) integra variables de comportamiento del consumidor, el ámbito empresarial y el área social.

Las variables de integración del modelo de Van Raaij se definen de la siguiente manera:

- Factores personales $(\mathrm{P})$ : actitud hacia el endeudamiento, edad, género.

- Ambiente percibido (E/P): percepción subjetiva de la situación financiera.

- Entorno económico (E): ingresos del grupo familiar y estado de endeudamiento.

- Comportamiento económico (B): hábitos y conductas de consumo. 
Figura 3

Variables de comportamiento del consumidor, el ámbito empresarial y el área social

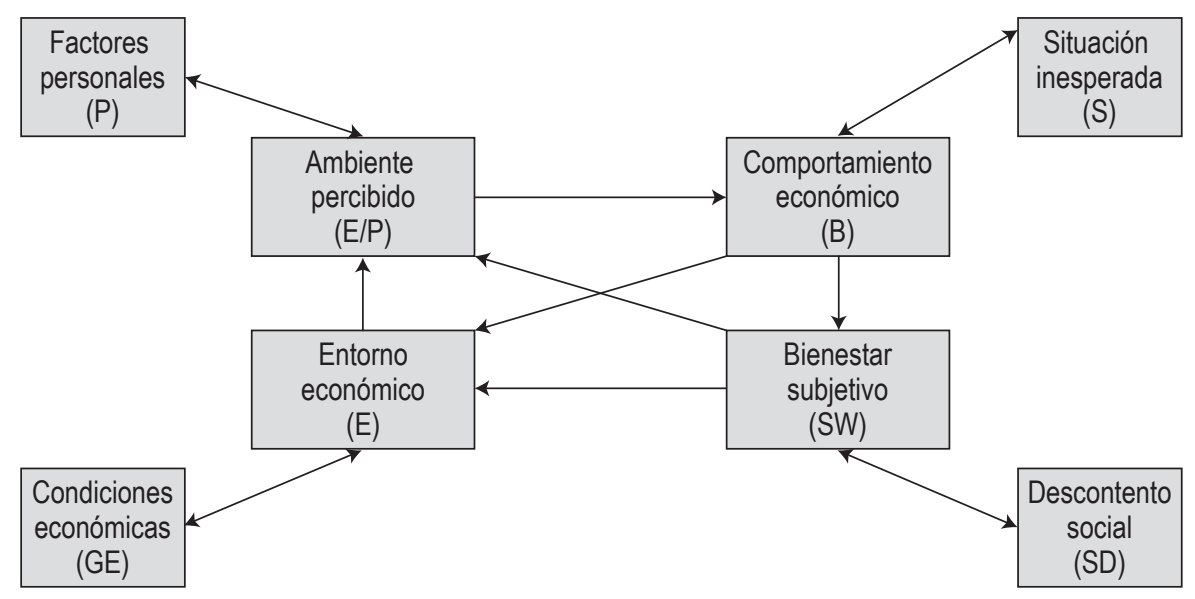

Elaboración propia.

- Bienestar subjetivo (SW).

- Situación inesperada (S).

- Condiciones económicas (GE).

- Descontento social (SD).

En el análisis realizado por los autores antes citados, la lógica del modelo se describe de la siguiente manera:

El mayor ingreso del grupo familiar sumado a un menor estado de endeudamiento (E) conduce a una percepción subjetiva de una situación financiera optimista (E/P), que a su vez influye sobre hábitos de consumo (B) de manera reflexiva; todo esto está asociado a un mayor bienestar subjetivo (SW), que a su vez deriva sobre el entorno económico (E) favorable, transformando al modelo en sistémico. La percepción subjetiva de la situación financiera más optimista incidirá sobre una actitud hacia el endeudamiento de manera austera, calificando al consumidor como eficiente y responsable. 
En sentido contrario, un menor nivel de ingresos familiares y menor estado de endeudamiento $(\mathrm{E})$ conduce a una percepción subjetiva $(\mathrm{E} / \mathrm{P})$ más pesimista, influyendo sobre los hábitos y conductas de consumo (B) de manera impulsiva; esta situación se asocia a un menor bienestar subjetivo (SW), que a su vez induce a un mayor estado de endeudamiento (E). La percepción subjetiva de una situación financiera pesimista influirá en una mayor inclinación o actitud irresponsable hacia el endeudamiento.

Este modelo, como lo manifiesta Denegri (2006) en su libro Introducción a la psicología económica, incorpora un aspecto de dinamismo que lo hace muy interesante. Permite múltiples combinaciones a la vez que puede servir de base de modelos más específicos que puedan ser integrados en este.

\section{Escala de actitud hacia el endeudamiento}

La medición de la actitud hacia el endeudamiento desarrollado por Denegri, Palavecinos y Ripoll (1998), citados por Rodríguez Vargas (2006) y Denegri (2006), operacionalmente se hace mediante la manifestación a las siguientes proposiciones en una escala tipo Likert:

1. El uso del crédito puede ser muy peligroso.

2. Es preferible tratar de pagar siempre al contado.

3. Es importante tratar de vivir de acuerdo al dinero que se tiene.

4. Si uno se lo propone, siempre se puede ahorrar algo de dinero.

5. Es importante pagar las deudas lo antes posible.

6. Hay que ser muy cuidadoso en el gasto del dinero.

7. La facilidad de obtener tarjetas de crédito es una causa del endeudamiento de la gente.

8. Usar el crédito permite tener una mejor calidad de vida.

9. Es una buena idea comprar algo ahora y pagarlo después.

10. El uso del crédito es una parte esencial del estilo de vida actual.

11. Pedir un préstamo es una muy buena idea. 


\section{Escala de hábitos y conductas de consumo}

El comportamiento económico, según Denegri (2006), se mide a través de la frecuencia con la que se manifiestan los consumidores con las siguientes preguntas, las mismas que han sido adaptadas al quehacer de un microempresario de la confección textil:

1. ¿Hace una lista de la materia prima e insumos que necesita comprar?

2. ¿Selecciona la materia prima e insumos según su calidad?

3. ¿Para distribuir su dinero, ordena los productos según su importancia antes de comprar?

4. ¿Selecciona la materia prima e insumos según su precio?

5. ¿Compara precios entre distintas marcas?

6. ¿Compara precios en distintos locales de venta?

7. ¿Lee las etiquetas de todos los productos antes de adquirir?

8. ¿Mira la cantidad, longitud o peso?

9. ¿Se asegura de que la empresa donde adquiere su maquinaria le ofrezca servicio técnico?

10. ¿Pregunta por el tiempo de garantía de los productos?

11. ¿Pregunta todas sus dudas al vendedor antes de comprar?

12. ¿Planifica todas sus compras?

13. ¿Cuando compra materia prima, pregunta o lee la etiqueta para saber características y cuidado que requiere?

14. ¿Examina detalladamente todos los productos que compra?

15. ¿Cree que gasta más de lo que debería?

16. ¿Compra en el comercio ambulante?

17. ¿Compra habitualmente a crédito?

18. Si solicita crédito, ¿pregunta por las tasas de interés y el incremento del precio final?

19. Si compra a crédito, ¿compara las tasas de interés en distintos locales? 


\section{Modelo psicoeconómico del consumidor}

Como lo señala Rodríguez Vargas (2006), la estructura del modelo psicoeconómico del consumidor ha sido diseñada desde una perspectiva más holística e integral, pero ante todo objetiva y medible, lo que facilita su posterior validación y aplicabilidad en cualquier entorno económico.

En el modelo planteado, las flechas directas indican una relación causal directa de un constructo a otro; así, en la zona de consumo el bienestar subjetivo se mide a través de los hábitos y conductas de consumo o simplemente la conducta económica es causa para el bienestar subjetivo; y este a su vez es causa del ingreso económico. En la misma zona de consumo, la línea sin flechas indica una correlación entre los constructos ingreso económico y conducta económica.

Figura 4

\section{Modelo psicoeconómico del consumidor}

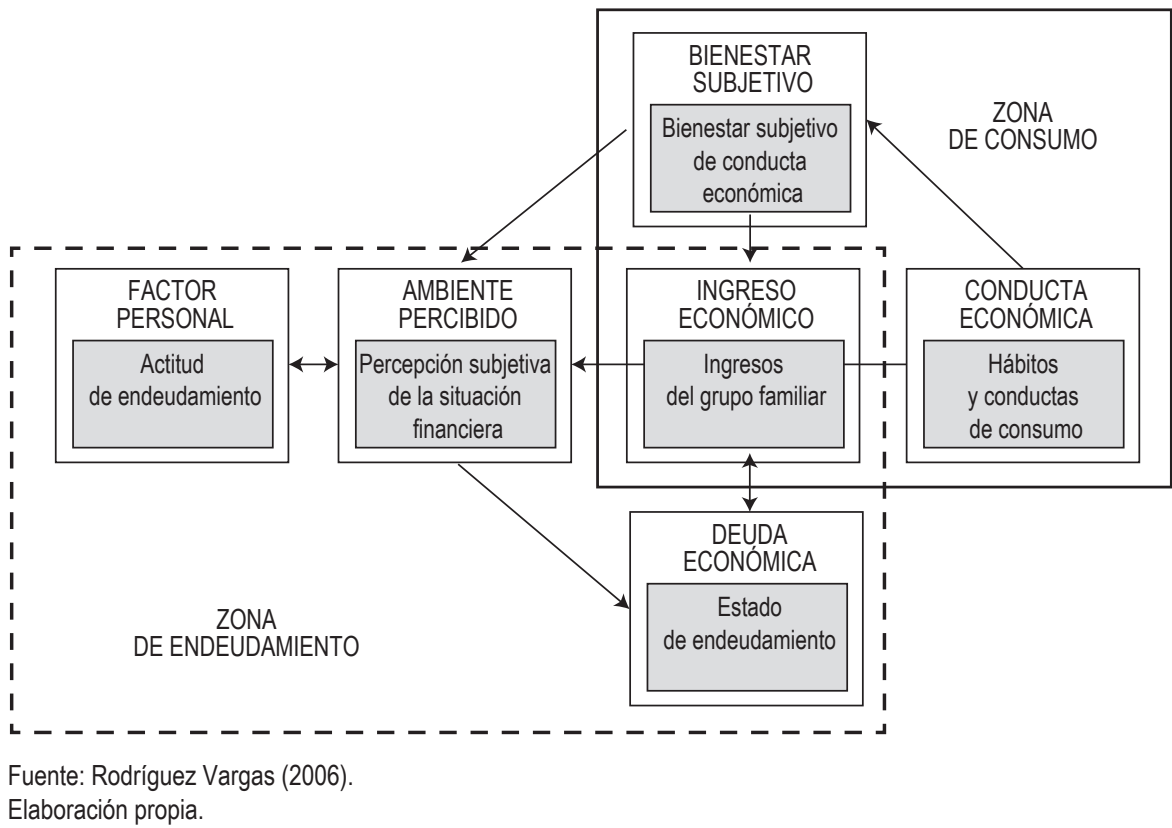


En la zona de endeudamiento, las líneas con doble flecha indican una relación recíproca entre los constructos; así, el factor personal, medido a través de la actitud hacia el endeudamiento, tiene una relación recíproca con el ambiente percibido, estimado a través de la percepción subjetiva de la situación financiera: difícil, regular, aceptable y buena. De igual manera, el ingreso económico y la deuda económica (estado de endeudamiento) tienen una relación recíproca. En la zona de endeudamiento, el ingreso económico tiene una relación causal con el ambiente percibido y este a su vez con el estado de endeudamiento.

Entre zonas de consumo y endeudamiento, el bienestar subjetivo es causa para el ambiente percibido.

Las relaciones causales descritas en el modelo de Rodríguez Vargas sirven de base para el análisis exploratorio mediante análisis multivariable de los datos que se obtengan de la encuesta diseñada para el efecto y aplicada a los clientes de las operadoras de microfinanzas.

\section{Estilo de vida y actitud ante las entidades financieras}

El conjunto de proposiciones planteada por Agell y Segarra (1997) está constituido por 21 frases de diferencial semántico para forzar al encuestado a manifestar de modo unívoco su acuerdo con una u otra frase; sin embargo, para concordar con las escalas de actitud de Denegri, Palavecinos y Ripo11 (1998) incluidas en el cuestionario usado en esta investigación, se han replanteado las proposiciones adaptándolas al objeto de investigación -microempresarios encuestados- sobre la base de una escala tipo Likert de 4 puntos. A continuación se exponen las proposiciones replanteadas:

1. Para cubrir mis necesidades de crédito es preferible trabajar con varias entidades financieras (bancos o cooperativas) que con una sola.

2. Las entidades financieras de microcrédito presentan grandes diferencias entre sí.

3. Es preferible conocer solo los servicios que se utiliza y no la mayoría que la entidad financiera ofrece.

4. Se debe controlar siempre los estados de cuenta que envían las entidades financieras. 
5. Uno debe ir al banco o cooperativa cuando no se tiene otra alternativa.

6. El banco o cooperativa me interesa sobre todo para hacer transacciones.

7. Las entidades financieras modernas y automatizadas dificultan a la hora de revisar los estados financieros o realizar una transacción.

8. Es preferible el uso de un cajero automático a la atención personal.

9. Es preferible desplazarse a la entidad financiera para resolver los asuntos personalmente antes que hacerlo por teléfono.

10. Si un banco o cooperativa ofrece mayor interés por los ahorros, debería cambiar de banco.

11. Si necesitase un crédito, solicitaría a mi banco o cooperativa habitual sin consultar a otros.

12. En una inversión, es más importante la seguridad que la rentabilidad.

13. En el momento de invertir, prefiero tomar personalmente mis decisiones antes que seguir el consejo de entendidos.

14. Lo más importante del servicio bancario o cooperativo es el trato y la atención prestados a los clientes antes que el interés que cobran.

15. En un negocio siempre es posible ahorrar.

16. Si no se dispone del dinero suficiente para comprar algo, se debe solicitar un crédito.

17. Es preferible pagar al contado que pagar a plazos.

18. Si se hace una inversión es preferible hacerlo a largo plazo antes que a corto plazo.

\section{Modelo metodológico}

El presente estudio, realizado bajo un enfoque descriptivo y explicativo, tiene como objetivo principal plantear una alternativa de caracterización del mercado microfinanciero -enfocado en la industria de la confección textilcomo referente metodológico que podría servir en el futuro para el estudio de otros sectores microempresariales. 
El método general de investigación escogido es el hipotético-deductivo, surgiendo, sobre esa base, el planteamiento de las siguientes hipótesis investigativas:

1. El uso de herramientas de análisis multivariable, por sector microempresarial, permitirá desarrollar una metodología para caracterizar y profundizar en el conocimiento del mercado microempresarial de la microindustria de la confección textil.

2. Los modelos planteados para el estudio del comportamiento del consumidor y validado en mercados de consumidores finales, pueden ser aplicables en el mercado microempresarial.

3. La percepción que los microempresarios tienen de las entidades financieras está relacionada con sus estilos de vida y actitud hacia las mismas.

\section{Técnicas para captura de datos}

Al momento de la investigación de campo se escogió, como técnica para la captura de datos, a la encuesta, la cual se basó en el diseño de un cuestionario estructurado -aplicado a los microempresarios de la confección textilque tuvo como referente la adaptación de las proposiciones descritas en los modelos psicoeconómicos del enfoque teórico y modificadas a una escala Likert de cuatro puntos.

El cuestionario, una vez probado -antes de su aplicación definitiva- fue aplicado a través de la realización de visitas personales a los microempresarios; siendo sus focos de captura de datos 66 variables ordenadas de la siguiente manera:

1. Las dos primeras preguntas se orientan a la identificación de la entidad operadora de microcrédito y el lugar donde se realiza la encuesta.

2. El primer bloque de proposiciones se orienta a medir la actitud de los microempresario hacia el endeudamiento mediante una escala de Likert de cuatro puntos.

3. En el segundo bloque se mide el estado de endeudamiento y la percepción de la situación financiera. 
4. El tercer bloque pretende medir el estilo de vida desde el punto de vista financiero y la actitud de los microempresarios hacia las entidades financieras, mediante afirmaciones que son evaluadas con una escala de Likert de cuatro puntos y una valoración que dan los microempresarios a las características y/o atributos de las operadoras de microcrédito.

5. A continuación se tiene un bloque de preguntas para medir los hábitos y conductas de consumo de los microempresarios en la compra de materia prima e insumos.

6. En los dos últimos bloques, las preguntas se orientan a medir las variables de estilo de vida cotidiana y variables demográficas.

En cuanto al diseño, selección de la muestra y recolección de datos se deben resaltar las siguientes premisas investigativas:

- Unidades de muestreo: microempresarios que mantienen una cuenta o crédito con la entidad financiera colaboradora.

- Alcance: ciudades de la región centro norte de la Sierra ecuatoriana (Ibarra, Atuntaqui, Otavalo, Quito, Latacunga, Ambato, Pelileo y Riobamba).

- Tamaño de la muestra: por las disponibilidades de tiempo, recursos y concentración de microempresarios de la confección textil en ciudades pequeñas, se optó por una muestra probabilística de tamaño 202, que para un nivel de confianza del $95,5 \%$ produce un error, en estado de incertidumbre, de un $7 \%$.

- Sistema de muestreo: el detalle de la muestra, escogida en cada ciudad, se hizo mediante muestreo aleatorio simple, de acuerdo al marco muestral proporcionado por la entidad financiera auspiciante de la investigación.

- Trabajo de campo: el proceso de recolección de datos se realizó con la participación de los asesores de crédito de una entidad financiera colaboradora del presente trabajo de investigación.

\section{Métodos para el análisis de datos}

Los métodos de análisis de datos utilizados en la presente investigación son: tabulaciones cruzadas (tablas de contingencia con prueba Ji cuadrada), análisis estadístico multivariable mediante técnicas tales como análisis fac- 
torial (método de componentes principales), análisis de conglomerados (algoritmo k-medias) y análisis discriminante múltiple.

Finalmente, se debe resaltar que todo el procesamiento y análisis de los datos capturados se hizo con el soporte del programa informático DYANE versión $4 .^{3}$

\section{Descripción de hallazgos y discusión de resultados}

\section{Actitud hacia el endeudamiento}

De los resultados del análisis de los principales componentes de las variables que miden la actitud hacia el endeudamiento, se observó que la matriz de coeficientes de correlación simple de las once variables investigadas, mediante el test de Bartlett, se rechaza la hipótesis nula de que la matriz de coeficientes de correlación no es significativamente distinta de una matriz de identidad para un nivel de significancia del $5 \%$, por lo que es pertinente la factorización.

Mediante la retención de tres factores y la aplicación de la rotación VARIMAX ${ }^{4}$ se obtuvieron las ecuaciones que se muestra a continuación, en donde cada factor, es una combinación lineal de las once variables que miden la actitud hacia el endeudamiento.

Factor $1=-0,1876 \mathrm{X} 1+0,1716 \mathrm{X} 2-0,0075 \mathrm{X} 3+0,0118 \mathrm{X} 4+0,1315 \mathrm{X} 5$

$+0,1641 \mathrm{X} 6+0,0492 \mathrm{X} 7+0,7733 \mathrm{X} 8+0,5467 \mathrm{X} 9+0,6889 \mathrm{X} 10+$ $0,8034 \mathrm{X} 11$

Factor $2=-0,0548 \mathrm{X} 1+0,2440 \mathrm{X} 2+0,6244 \mathrm{X} 3+0,5116 \mathrm{X} 4+0,7568 \times 5$

$+0,7098 \times 6+0,2122 \times 7+0,1586 \times 8-0,2005 X 9+0,2433 \times 10+$ $0,0333 \mathrm{X} 11$

Factor $3=0,6760 \mathrm{X} 1+0,6579 \mathrm{X} 2+0,2144 \mathrm{X} 3+0,1285 \mathrm{X} 4+0,0287 \mathrm{X} 5-$ $0,0011 \mathrm{X} 6+0,4731 \mathrm{X} 7+0,0145 \mathrm{X} 8+0,3529 \mathrm{X} 9-0,0619 \mathrm{X} 10-0,0525 \mathrm{X} 11$

\footnotetext{
3. Software adjunto al texto "Diseño y análisis de encuestas en investigación social y de mercados", cuyo autor es Miguel Santesmases Mestre.

4. Rotación ortogonal de los factores retenidos con el fin de facilitar la interpretación de su resultado.
} 
Las variables que mayor carga tienen con los diferentes factores se describen en la tabla 2:

Tabla 2

Variables que miden la actitud hacia el endeudamiento

\begin{tabular}{|c|c|c|c|}
\hline & Variables & $\begin{array}{c}\text { Carga } \\
\text { del factor }\end{array}$ & $\begin{array}{l}\text { Actitud } \\
\text { evaluada }\end{array}$ \\
\hline \multirow{4}{*}{ Factor 1} & $\begin{array}{l}\text { X8 - Usar el crédito permite tener } \\
\text { una mejor calidad de vida. }\end{array}$ & 0,7733 & \multirow{4}{*}{$\begin{array}{l}\text { Actitud propensa } \\
\text { al endeudamiento. }\end{array}$} \\
\hline & $\begin{array}{l}\text { X9 - Es una buena idea comprar } \\
\text { algo ahora y pagarlo después. }\end{array}$ & 0,5467 & \\
\hline & $\begin{array}{l}\text { X10 - El uso del crédito es una parte } \\
\text { esencial del estilo de vida actual. }\end{array}$ & 0,6889 & \\
\hline & $\begin{array}{l}\text { X11 - Pedir un préstamo } \\
\text { es una muy buena idea. }\end{array}$ & 0,8034 & \\
\hline \multirow{4}{*}{ Factor 2} & $\begin{array}{l}\text { X3 - Es importante tratar de vivir } \\
\text { de acuerdo al dinero que se tiene. }\end{array}$ & 0,6244 & \multirow{4}{*}{$\begin{array}{l}\text { Cuidador de } \\
\text { la economía. }\end{array}$} \\
\hline & $\begin{array}{l}\text { X4 - Si uno se lo propone, siempre } \\
\text { puede ahorrar algo de dinero. }\end{array}$ & 0,5116 & \\
\hline & $\begin{array}{l}\text { X5 - Es importante pagar las deudas } \\
\text { lo antes posible. }\end{array}$ & 0,7568 & \\
\hline & $\begin{array}{l}\text { X6 - Hay que ser muy cuidadoso } \\
\text { en el gasto del dinero. }\end{array}$ & 0,7098 & \\
\hline \multirow{3}{*}{ Factor 3} & $\begin{array}{l}\text { X1 - El uso del crédito puede ser } \\
\text { muy peligroso. }\end{array}$ & 0,6760 & \multirow{3}{*}{$\begin{array}{l}\text { Actitud de temor } \\
\text { al endeudamiento. }\end{array}$} \\
\hline & $\begin{array}{l}\text { X2 - Es preferible tratar de pagar } \\
\text { siempre al contado. }\end{array}$ & 0,6579 & \\
\hline & $\begin{array}{l}\text { X7 - La facilidad de obtener tarjetas } \\
\text { de crédito es una causa } \\
\text { del endeudamiento de la gente. }\end{array}$ & 0,4731 & \\
\hline
\end{tabular}

Fuente: estudio de campo.

Elaboración propia. 
A criterio del investigador, los nombres que describen a estos factores por la asociación con las variables son: actitud propensa al endeudamiento, cuidador de la economía y actitud de temor al endeudamiento, respectivamente.

Con estas nuevas variables y mediante el algoritmo k-medias, se ha dividido a la actitud al endeudamiento en tres grupos; sin embargo, es preciso aclarar que el modelo de K-medias tiene como objetivo obtener $\mathrm{K}$ grupos (clusters) de tal manera que la suma de cuadrados entre los valores de las variables observadas en cada individuo de la muestra respecto de los valores medios del grupo al que pertenece sea mínima (Santesmases Mestre 2009). En la elección del número de conglomerados no existen reglas exactas; sin embargo, Agell y Segarra (1997) manifiestan que una regla empírica para determinar el número de grupos aconseja un mínimo de tres y un máximo de siete; según Malhotra (2004), los tamaños relativos de los conglomerados deben ser significativos.

Los grupos quedan definidos como se muestra en la siguiente tabla:

Tabla 3

Grupos de factores por la asociación con las variables

\begin{tabular}{|l|c|c|}
\hline \multicolumn{1}{|c|}{ Grupo } & Individuos & Porcentaje \\
\hline Actitud contraria al endeudamiento & 61 & $30,8 \%$ \\
\hline Actitud de endeudamiento responsable & 68 & $34,3 \%$ \\
\hline Actitud de comportamiento difuso & 69 & $34,9 \%$ \\
\hline Total & 198 & $100,0 \%$ \\
\hline
\end{tabular}

Fuente: estudio de campo.

Elaboración propia.

\section{Estilo de vida y actitud hacia las entidades financieras}

Del análisis de componentes principales practicado a las 18 variables que miden el estilo de vida y actitud hacia las entidades financieras, se muestra a continuación, en la tabla 4, el factor, la variable y el enfoque: 
Tabla 4

Estilo de vida y actitud hacia las entidades financieras

\begin{tabular}{|c|c|c|c|}
\hline Factor & Variable & Carga factorial & Enfoque \\
\hline \multirow{3}{*}{ Factor 1} & $\begin{array}{l}\text { Y4 - Se debe controlar siempre los estados } \\
\text { de cuenta que envían las entidades financieras. }\end{array}$ & $0,6670^{*}$ & \multirow{3}{*}{$\begin{array}{l}\text { Enfoque } \\
\text { a la prolijidad y } \\
\text { el ahorro. }\end{array}$} \\
\hline & $\begin{array}{l}\text { Y14 - Lo más importante del servicio bancario } \\
\text { o cooperativo es el trato y la atención prestados a } \\
\text { los clientes antes que el interés que cobran. }\end{array}$ & $0,5728^{*}$ & \\
\hline & Y15 - En un negocio siempre es posible ahorrar. & $0,6402^{*}$ & \\
\hline \multirow{7}{*}{ Factor 2} & $\begin{array}{l}\text { Y1 - Para cubrir mis necesidades de crédito } \\
\text { es preferible trabajar con varias entidades } \\
\text { financieras (bancos o cooperativas) } \\
\text { que con uno solo. }\end{array}$ & $0,4185^{*}$ & \multirow{7}{*}{$\begin{array}{l}\text { Enfoque a la } \\
\text { modernidad } \\
\text { y finanzas. }\end{array}$} \\
\hline & $\begin{array}{l}\text { Y3 - Es preferible conocer solo los servicios } \\
\text { que se utiliza y no la mayoría que la entidad } \\
\text { financiera ofrece. }\end{array}$ & $0,6873^{*}$ & \\
\hline & $\begin{array}{l}\text { Y5 - Uno debe ir al banco o cooperativa cuando no } \\
\text { se tiene otra alternativa. }\end{array}$ & $0,5797^{*}$ & \\
\hline & $\begin{array}{l}\text { Y6 - El banco o cooperativa me interesa } \\
\text { sobre todo para hacer transacciones. }\end{array}$ & $0,3804^{*}$ & \\
\hline & $\begin{array}{l}\text { Y8 - Es preferible el uso de un cajero automático a } \\
\text { la atención personal. }\end{array}$ & $0,5354^{*}$ & \\
\hline & $\begin{array}{l}\text { Y11 - Si necesitase un crédito, solicitaría } \\
\text { a mi banco o cooperativa habitual } \\
\text { sin consultar a otros. }\end{array}$ & $0,3578^{*}$ & \\
\hline & $\begin{array}{l}\text { Y18 - Si se hace una inversión es preferible } \\
\text { hacerlo a largo plazo antes que a corto plazo. }\end{array}$ & $0,3290^{*}$ & \\
\hline \multirow{3}{*}{ Factor 3} & $\begin{array}{l}\text { Y7 - Las entidades financieras modernas } \\
\text { y automatizadas dificultan a la hora de revisar } \\
\text { los estados financieros o realizar una transacción. }\end{array}$ & $0,4956^{*}$ & \multirow{3}{*}{$\begin{array}{l}\text { Enfoque a la } \\
\text { seguridad. }\end{array}$} \\
\hline & $\begin{array}{l}\text { Y12 - En una inversión, es más importante } \\
\text { la seguridad que la rentabilidad. }\end{array}$ & $0,5424^{*}$ & \\
\hline & $\begin{array}{l}\text { Y13 - En el momento de invertir, prefiero tomar } \\
\text { personalmente mis decisiones antes que seguir } \\
\text { el consejo de entendidos. }\end{array}$ & $0,7403^{*}$ & \\
\hline
\end{tabular}




\begin{tabular}{|c|c|c|c|}
\hline \multirow{2}{*}{ Factor 4} & \begin{tabular}{|l} 
Y16 - Si no se dispone del dinero suficiente \\
para comprar algo, se debe solicitar un crédito.
\end{tabular} & $-0,4929^{*}$ & \multirow{2}{*}{$\begin{array}{l}\text { Enfoque } \\
\text { a la austeridad }\end{array}$} \\
\hline & $\begin{array}{l}\text { Y17 - Es preferible pagar al contado } \\
\text { que pagar a plazos. }\end{array}$ & $31^{*}$ & \\
\hline \multirow{3}{*}{ Factor 5} & $\begin{array}{l}\text { Y2 - Las entidades financieras de microcrédito } \\
\text { presentan grandes diferencias entre sí. }\end{array}$ & $0,6161^{*}$ & \multirow{3}{*}{$\begin{array}{l}\text { Enfoque } \\
\text { tradicionalista } \\
\text { y buscador de } \\
\text { conveniencia. }\end{array}$} \\
\hline & $\begin{array}{l}\text { Y9 - Es preferible desplazarse a la entidad } \\
\text { financiera para resolver los asuntos personalmente } \\
\text { antes que hacerlo por teléfono. }\end{array}$ & $0,6283^{*}$ & \\
\hline & $\begin{array}{l}\text { Y10 - Si un banco o cooperativa ofrece mayor } \\
\text { interés por los ahorros, debería cambiar de banco. }\end{array}$ & $0,5080^{*}$ & \\
\hline
\end{tabular}

Fuente: estudio de campo.

Elaboración propia.

Con los factores obtenidos, se procedió a caracterizar el estilo de vida y actitud hacia las entidades financieras en cinco grupos, de la siguiente manera:

Grupo 1: Constituido por 34 encuestados $(17,17 \%)$ está caracterizado positivamente por los factores 1 y 5 , y negativamente por el factor 4 , de la siguiente manera: aunque no demuestran austeridad, son prolijos con sus estados de cuenta y con tendencia al ahorro; por otro lado, se muestran tradicionalistas y buscadores de conveniencias para sus intereses.

Grupo 2: Este grupo está caracterizado en forma negativa por el factor 5, y de acuerdo a las variables que agrupa, su tipología es la siguiente: se muestran leales a su entidad financiera (no son buscadores de conveniencia); y no parecen ser de comportamiento tradicionalista, más bien podría interpretarse como prácticos. El grupo está constituido por 29 encuestados (14,65\%).

Grupo 3: El grupo, formado por 47 investigados (23,74\%), se encuentra caracterizado positivamente por los factores 2, 3 y 4, describiéndose como: individuos con enfoque moderno y mentalidad financiera; buscan seguridad en sus inversiones y con tendencia a la austeridad.

Grupo 4: El factor que mejor resume a este grupo es el factor 4, aunque en forma negativa; por consiguiente, la característica de este grupo es: prefieren trabajar con una sola entidad financiera; aunque les interesa conocer la mayoría de servicios que se ofrece, tienen tendencia solo por las transaccio- 
nes; son proclives a la atención personal y prefieren las inversiones a corto plazo. Este grupo está constituido por 52 encuestados $(26,26 \%)$.

Grupo 5: Los factores 1 y 3 influyen negativamente sobre la característica de este grupo, los mismos que se describen como: no son prolijos en el control de los estados de cuenta; dan mayor importancia a los intereses que cobran por sobre la seguridad y la atención y tienen tendencia a dejarse asesorar financieramente. El grupo lo constituyen 36 personas (18,18\%).

\section{Hábitos y conductas de consumo}

Mediante el análisis de componentes principales, practicado al constructo que mide los hábitos y conductas de consumo de los microempresarios de la confección textil, se obtuvieron los siguientes resultados:

Tabla 5

Hábitos y conductas de consumo de los microempresarios de la confección textil

\begin{tabular}{|c|c|c|c|}
\hline Factor & Variables & Carga factorial & Enfoque \\
\hline \multirow{6}{*}{ Factor 1} & $\begin{array}{l}\mathrm{Z1} \text { - ¿Hace una lista de la materia prima } \\
\text { e insumos que se necesita comprar? }\end{array}$ & $0,6738^{*}$ & \multirow{6}{*}{$\begin{array}{l}\text { Enfoque } \\
\text { a la planificación }\end{array}$} \\
\hline & $\begin{array}{l}\text { Z2 - ¿Selecciona la materia prima } \\
\text { e insumos que adquiere según su calidad? }\end{array}$ & $0,7596^{*}$ & \\
\hline & $\begin{array}{l}\text { Z3 - Para distribuir el dinero, ¿ordena } \\
\text { los productos según su importancia } \\
\text { antes de comprar? }\end{array}$ & $0,7798^{*}$ & \\
\hline & $\begin{array}{l}\text { Z4 - ¿Selecciona la materia prima } \\
\text { e insumos según sus precios? }\end{array}$ & $0,5898^{*}$ & \\
\hline & Z12 - ¿Planifica todas sus compras? & $0,7090^{*}$ & \\
\hline & $\begin{array}{l}\text { Z14 - ¿Examina detalladamente } \\
\text { todos los productos que compra? }\end{array}$ & $0,5205^{\star}$ & \\
\hline
\end{tabular}




\begin{tabular}{|c|c|c|c|}
\hline \multirow{3}{*}{ Factor 2} & Z17 - ¿Compra habitualmente a crédito? & $0,5295^{*}$ & \multirow{3}{*}{$\begin{array}{l}\text { Uso del crédito } \\
\text { en forma selectiva }\end{array}$} \\
\hline & $\begin{array}{l}\text { Z18 - Si solicita crédito, ¿pregunta } \\
\text { por las tasas de interés y el incremento } \\
\text { del precio final? }\end{array}$ & $0,7907^{*}$ & \\
\hline & $\begin{array}{l}\text { Z19 - Si compra a crédito, ¿compara } \\
\text { las tasas de interés en distintos locales? }\end{array}$ & $0,7921^{*}$ & \\
\hline \multirow{2}{*}{ Factor 3} & $\begin{array}{l}\text { Z15 - ¿Cree que gasta más } \\
\text { de lo que debería? }\end{array}$ & $0,7540^{*}$ & \multirow{2}{*}{$\begin{array}{l}\text { Hábitos } \\
\text { y conductas } \\
\text { de comparación } \\
\text { informal. }\end{array}$} \\
\hline & Z16 - ¿Compra en el comercio ambulante? & $0,7582^{*}$ & \\
\hline \multirow{2}{*}{ Factor 4} & $\begin{array}{l}Z 5-¿ \text { ¿Compara precios } \\
\text { entre distintas marcas? }\end{array}$ & $0,8763^{*}$ & \multirow{2}{*}{$\begin{array}{l}\text { Enfoque } \\
\text { en los precios. }\end{array}$} \\
\hline & $\begin{array}{l}\text { Z6 - ¿Compara los precios } \\
\text { en distintos locales de venta? }\end{array}$ & $0,7918^{*}$ & \\
\hline \multirow{6}{*}{ Factor 5} & $\begin{array}{l}\text { Z7 - ¿Lee las etiquetas de todos } \\
\text { los productos antes de adquirir? }\end{array}$ & $0,6141^{*}$ & \multirow{6}{*}{$\begin{array}{l}\text { Enfoque } \\
\text { en la calidad. }\end{array}$} \\
\hline & Z8 - ¿Mira la cantidad, longitud o peso? & $0,5220^{*}$ & \\
\hline & $\begin{array}{l}\text { Z9 - ¿Se asegura de que la maquinaria } \\
\text { tenga servicio técnico? }\end{array}$ & $0,7453^{*}$ & \\
\hline & $\begin{array}{l}\text { Z10 - ¿Pregunta por el tiempo de garantía } \\
\text { de los productos? }\end{array}$ & $0,7208^{*}$ & \\
\hline & $\begin{array}{l}\text { Z11 - ¿Pregunta todas sus dudas } \\
\text { al vendedor antes de comprar? }\end{array}$ & $0,6514^{*}$ & \\
\hline & $\begin{array}{l}\text { Z12 - Cuando compra materia prima } \\
\text { ¿pregunta o lee la etiqueta para saber } \\
\text { características y cuidados que requiere? }\end{array}$ & $0,5863^{*}$ & \\
\hline
\end{tabular}

Fuente: estudio de campo.

Elaboración propia.

La asociación de las variables en los diferentes factores subyacentes ha permitido definir, a criterio de los autores, los enfoques de cada uno de ellos. En la división de grupos con características diferentes en términos de hábitos y conductas de consumo, practicado a los cinco factores mediante el algoritmo de k-medias, se ha definido lo siguiente: 
Grupo 1: Los factores que caracterizan a este grupo y de forma negativa son el factor $1 \mathrm{y}$ factor 4 , quedando la descripción de la siguiente manera: no son planificadores de sus compras y no hacen diferenciación con los precios de las materias primas e insumos con respecto a las marcas y puntos de venta. El número de individuos pertenecientes al grupo es de $36(18,2 \%)$.

Grupo 2: Este grupo, constituido por 86 encuestados $(43,4 \%)$, está caracterizado negativamente por el factor 3 y positivamente por el factor 5 , quedando de la siguiente manera: las compras lo hacen habitualmente en el comercio formal y consideran que no gastan más de lo que deberían; y la adquisición de la materia prima e insumos está enfocado a la calidad.

Grupo 3: En este grupo, tienen mayor influencia positiva los factores 2 y 3, los mismos que describen a $37(18,7 \%)$ de la muestra como: compran habitualmente a crédito y son selectivos con el precio final; son proclives a la compra en el comercio informal, una posible causa es que "creen que gastan más de lo que deberían".

Grupo 4: El único factor y que caracteriza en forma negativa a 20 investigados $(10,1 \%)$ es el factor 5 , quedando de la siguiente manera: en la compra de materia prima e insumos no se enfocan en la calidad de los mismos.

Grupo 5: La característica del más pequeño de los grupos, formado por $19(9,6 \%)$, está influido por los factores 1 y 4 positivamente y 2 en forma negativa. La tipología de este grupo es la siguiente: son planificadores de sus compras; no hacen uso del crédito en las compras; y la compra se enfoca en los precios.

\section{Análisis de grupos (Cluster analysis)}

Mediante esta técnica se pretende dividir a la muestra de estudio en distintos grupos internamente homogéneos, pero distintos entre sí, conservándose las variables originales sin el paso previo de factorización. El método ha sido aplicado a los diferentes constructos tales como: actitud al endeudamiento, estilo de vida y actitud hacia las entidades financieras, y hábitos y conductas de consumo. 


\section{Interpretación de la actitud hacia el endeudamiento}

Considerando los tamaños relativos de los grupos y la interpretación de la asociación de las proposiciones, se ha caracterizado a la actitud al endeudamiento tal como se describe a continuación, con la aclaración de que las variables X4, X5 y X6 no discriminan mayormente entre los grupos, por tener un rango entre el mayor y menor promedio de cada variable entre grupos relativamente pequeños:

Grupo 1: Este segmento, que constituye el 23,23\% de los investigados, presenta las siguientes características: piensan que el uso del crédito no es muy peligroso (X1); la facilidad de obtener tarjetas de crédito no es causa fundamental del endeudamiento de la gente (X7); y tienen tendencia a pagar a plazos (X2). Un nombre asociado a la tipología de este grupo se ha definido como "actitud positiva al endeudamiento con riesgo de sobreendeudamiento".

Grupo 2: Aunque tienen preferencia por pagar siempre al contado (X2), este segmento se caracteriza por lo siguiente: consideran que el crédito es parte esencial del estilo de vida y permite mejorar la calidad de vida (X10, $\mathrm{X} 8$ ); se muestran proclives al endeudamiento, tal como lo demuestran las puntuaciones de los valores medios de las variables X9 y X11. La frase que mejor se adapta al grupo es de "actitud positiva al endeudamiento".

Grupo 3: E1 27,27\% de los encuestados presentan las siguientes características: sostienen que la facilidad en la obtención de tarjetas de crédito es causa del endeudamiento (X7) y tratan de vivir con el dinero que se tiene (X3). A este grupo se lo ha definido como de "actitud negativa al endeudamiento".

\section{Interpretación del estilo de vida y actitud hacia las entidades financieras}

Bajo el mismo patrón de análisis de la actitud al endeudamiento y considerando el número de variables que miden la actitud hacia las entidades financieras, se ha dividido a la muestra de estudio en cinco grupos. Las características que presentan estos cinco grupos son: 
Grupo 1: Los atributos más relevantes de este segmento (10,1\% respecto a la muestra), son: alto grado de preferencia por la atención personalizada (Y8) y, por consiguiente, el trato, la atención y la seguridad prestada por la entidad financiera tienen mayor importancia que los intereses que cobran (Y8, Y14 y Y12); para cubrir las necesidades de crédito, prefieren trabajar con una sola entidad financiera (lealtad a la entidad) (Y1); son prolijos en el control de los estados de cuenta (Y4); y si no disponen de dinero suficiente para comprar algo, no dudarían en solicitar un crédito. El nombre asignado a este grupo es "lealtad y prolijidad".

Grupo 2: A este grupo, que constituye el $16,67 \%$, se lo puede describir de la siguiente manera: tienen preferencia por conocer todos los servicios que la entidad financiera ofrece (Y3); tienen tendencia a ir a la entidad financiera (Y5); consideran que es preferible desplazarse a la entidad financiera para resolver los asuntos personalmente antes que hacerlo por teléfono (Y9); y tienen tendencia a la inversión a corto plazo (Y18). Las características están resumidas en un término: "tradicionalistas".

Grupo 3: La tipología de este grupo se define a continuación como: para cubrir las necesidades de crédito, prefieren trabajar con varios bancos o cooperativas que con uno solo (Y1); consideran que las entidades financieras de microcrédito presentan diferencias entre sí (Y2); se interesan solo por los servicios que utilizan y no por la mayoría que la entidad financiera ofrece (Y3); prefieren el uso de cajeros automáticos a la atención personal (Y8); sostienen que en un negocio siempre es posible ahorrar (Y15); tienen tendencia a pagar al contado que a plazos, sin embargo, si hacen una inversión, prefieren hacerlo a largo plazo (Y17, Y18). Este grupo constituye el 32,32\% y se le ha asignado el adjetivo de "instruidos y modernos".

Grupo 4: Al grupo 4 le corresponde el 17,68\% y presenta las siguientes características: piensan que deben ir al banco o cooperativa cuando no se tiene otra alternativa y les interesa la entidad financiera sobre todo para hacer transacciones (Y5, Y6); perciben que las entidades modernas y automatizadas dificultan a la hora de revisar los estados financieros o realizar una transacción (Y7); estarían dispuestos a cambiar de banco o cooperativa si otra entidad ofrece mayores intereses por sus ahorros (Y10); si necesitasen un crédito, solicitarían a su entidad financiera habitual sin consultar a otras (Y11) y en el momento de invertir prefieren tomar decisiones propias antes 
que seguir los consejos de entendidos (Y13). Se le ha asignado el calificativo de "buscadores de conveniencia".

Grupo 5: Las variables más importantes y que describen a este grupo, que tiene un porcentaje de $23,23 \%$ respecto a la muestra, son: en una inversión, tienen una leve tendencia a la seguridad que a la rentabilidad (Y12); al momento de invertir prefieren tomar las decisiones con asesoría de entendidos (Y13), y relativa preferencia al trato y atención prestado a los clientes antes que el interés que cobran (Y14). El nombre que mejor se ajusta a las características descritas es de "buscadores de seguridad".

\section{Interpretación de los hábitos y conductas de consumo}

El análisis del algoritmo k-medias practicado a las variables que miden los hábitos y conductas de consumo ha sido elaborado con tres y cinco grupos, respectivamente. Por el número de variables del constructo (19) se ha escogido la segunda (cinco grupos), con un porcentaje de variación explicada del 31,68\% y mayor afinidad entre las variables que lo conforman. Los porcentajes de cada grupo respecto al total de la muestra es de 16,16, 9,09, $10,61,34,85$ y $29,29 \%$, respectivamente.

Los cinco grupos quedan definidos de la siguiente manera:

Grupo 1: Las variables que más contribuyen a la caracterización de este grupo y, según los valores medios obtenidos, se puede describir de la siguiente manera: pocas veces hacen una lista de la materia prima e insumos que necesitan adquirir; desde el punto de vista de prioridades o necesidades más urgentes, pocas veces clasifican sus productos antes de la compra (variables Z1 y Z3); la afirmación anterior se corrobora con la escasa planificación de sus compras (Z12); en lo que respecta a la calidad, se evidencia la poca importancia que dan a la selección de la materia prima e insumos (Z2 y Z14). El nombre que recibe el grupo es de "no planificadores".

Grupo 2: Este grupo se caracteriza por el siguiente comportamiento de compra: cuando adquieren maquinaria o bienes de capital relacionados con su actividad, pocas veces se aseguran de que tengan servicio de mantenimiento, tiempo de garantía y dudas sobre el producto (Z9, Z10, Z11); antes de la adquisición de un producto, rara vez leen las etiquetas adjuntas al 
mismo (Z7); de igual manera sucede con la compra de materia prima para saber las características y cuidados que requieren (Z13) y, según los valores medios de las variables Z4, Z5, Z6 y Z8, no dan importancia a la selección de la materia prima e insumos según sus precios, por lo que rara vez comparan entre marcas y puntos de venta y eventualmente examinan la cantidad, longitud o peso de los productos. A este grupo se le ha asignado el nombre de "comportamiento irresponsable en la compra".

Grupo 3: Los hábitos de conducta y consumo de este grupo está caracterizado por lo siguiente: tienen tendencia a comprar a crédito (Z17); en la compra a crédito pocas veces preguntan por las tasas de interés y el precio final, así como la comparación en distintos puntos de venta (Z18 y Z19); para distribuir el dinero, siempre ordenan los productos según su importancia antes de la compra y con frecuencia comparan los precios entre distintas marcas (Z3 y Z5). El nombre que se acopla a sus características es de "uso responsable del crédito".

Grupo 4: En contraposición al grupo 1 y 2, la tipología de este grupo se describe como sigue: frecuentemente hacen un listado de la materia prima e insumos que necesitan adquirir y seleccionan según su calidad (Z1 y Z2); también es importante destacar que la selección de la materia prima e insumos se hacen sobre la base de la comparación de precios y en distintos puntos de venta (Z4 y Z6); para la adquisición de un producto, previamente, leen las etiquetas adjuntas y examinan la cantidad, longitud o peso de las mismas, así como la disponibilidad de servicio técnico y garantía (Z7, Z8, Z9 y Z10); cuando tienen dudas sobre el producto, siempre preguntan al vendedor (Z11); son planificadores de sus compras (Z12); frecuentemente se interesan por saber las características y cuidados que requieren la materia prima e insumos, y examinan en detalle los productos que compran (Z13 y Z14); cuando solicitan crédito, siempre preguntan por las tasas de interés y precio final, y comparan en distintos puntos de venta (Z18 y Z19); consideran que muy pocas veces gastan más de lo que deberían y por lo regular no compran en el comercio ambulante (Z15 y Z16). Este segmento, que constituye el más grande, ha sido sintetizado como "espíritu emprendedor con enfoque en la calidad y reflexivo en el uso del crédito". 
Grupo 5: A pesar de que tan solo dos variables caracterizan a este grupo, es necesario señalar que constituyen el $29,29 \%$ de la muestra y tienen la siguiente descripción: tendencia a comprar en el comercio ambulante y muy pocas veces adquieren sus productos a crédito. Se le ha asignado el calificativo de "hábitos y conducta de compra informal".

\section{Análisis de asociaciones/dependencias entre variables}

Mediante la técnica de análisis discriminante se pretende explicar la pertenencia de individuos a grupos preestablecidos con técnicas de análisis factorial y análisis de grupos, respectivamente, practicadas a las variables de actitud al endeudamiento, estilo de vida y actitud hacia las entidades financieras, y hábitos y conductas de consumo. Del mismo modo, se busca una relación entre los diferentes constructos investigados a través de la encuesta practicada.

\section{Actitud al endeudamiento}

En los resultados se muestran los autovalores: a) los coeficientes estandarizados de las funciones discriminantes canónicas con sus respectivas ecuaciones discriminantes, b) las correlaciones entre las variables y las funciones discriminantes, c) los valores de las funciones en los centroides de los grupos, d) la matriz de confusión, e) para un nivel de significancia del 5\%, se validó el modelo aplicado.

En la matriz de confusión e) el porcentaje de las asignaciones acertadas por las funciones discriminantes es de $96,46 \%$, quedando los grupos según las funciones discriminantes como: 
Tabla 6

Grupos según las funciones discriminantes

\begin{tabular}{|l|c|c|}
\hline \multicolumn{1}{|c|}{ Grupos } & Reasignación & Porcentaje \\
\hline $\begin{array}{l}\text { Grupo 1 } \\
\text { (Actitud contraria al endeudamiento) }\end{array}$ & 63 & $30,8 \%$ \\
\hline $\begin{array}{l}\text { Grupo 2 } \\
\text { (Actitud de endeudamiento responsable) }\end{array}$ & 64 & $32,3 \%$ \\
\hline $\begin{array}{l}\text { Grupo 3 } \\
\text { (Actitud de comportamiento difuso) }\end{array}$ & 71 & $35,9 \%$ \\
\hline Total & 198 & $100,0 \%$ \\
\hline
\end{tabular}

Fuente: estudio de campo.

Elaboración propia.

Similar análisis se realizó con las tres categorías obtenidas a través del algoritmo k-medias y las once variables que miden la actitud hacia el endeudamiento, obteniéndose una matriz de confusión con $95,45 \%$ de asignación correcta.

\section{Relación entre la percepción subjetiva de la situación financiera y la actitud hacia el endeudamiento}

Considerando que la variable X13, la cual mide la percepción subjetiva de la situación financiera de los investigados, contiene pocos individuos en las categorías extremas "difícil" con 6 y "muy bien" con 13, se ha procedido a una reclasificación de las categorías, quedando en: "mala o regular" con 55 y "buena o muy buena" con 143 .

Con esta nueva categorización, se ha realizado una tabulación cruzada de datos entre la percepción subjetiva de la situación financiera del negocio y la actitud hacia el endeudamiento resumido en tres categorías mediante análisis de componentes principales algoritmo k-medias. En los resultados se pudo observar que a un nivel de significación del 6\%, existe una dependencia entre 
la percepción subjetiva y la actitud hacia el endeudamiento; esto es, cuando la percepción de la situación financiera es mala o regular, la actitud contraria al endeudamiento es mayor.

El mismo análisis se hizo entre la percepción subjetiva y los grupos definidos mediante el algoritmo k-medias. En los resultados se observó que a un nivel de significación del $6 \%$ existe una independencia entre las variables analizadas $(\mathrm{p}=0,2203)$.

\section{Relación entre la percepción subjetiva de la situación financiera y el bienestar subjetivo de la conducta económica}

Mediante tabulación cruzada entre la percepción subjetiva de la situación financiera manifestada por los investigados y el bienestar subjetivo medido a través de los hábitos y conductas de consumo mediante análisis de componentes principales practicado a 19 variables, se infirió que a un nivel de significancia del $1 \%$ existe una dependencia entre las variables analizadas (Ji cuadrado con 4 grados de libertad $=14,8463$ y probabilidad $\mathrm{p}=0,0050$ ).

Similar análisis se ha realizado con la percepción subjetiva de la situación financiera y el bienestar subjetivo medido a través de los hábitos y conductas de consumo mediante el algoritmo k-medias. De los resultados obtenidos, se deduce que las variables analizadas son dependientes a un nivel de significación del 5\% $(\mathrm{p}=0,0141)$.

\section{Relación entre los ingresos económicos del grupo familiar y conducta económica}

Mediante una tabulación cruzada de la variable que mide el ingreso económico del grupo familiar y la conducta económica determinada mediante análisis de componentes principales y el algoritmo k-medias, se observó que existe una dependencia entre estas dos variables a un nivel de significación del 5\% cuando la metodología de división en grupos ha sido hecha con análisis de componentes principales previo; en tanto que al usar la clasificación 
solo con el algoritmo k-medias se evidencia una independencia entre variables a un nivel de significancia del $5 \%$.

\section{Relación entre la percepción subjetiva de la situación financiera y el ingreso económico familiar}

Según los resultados, con un nivel de significancia del 5\% se evidencia una relación de dependencia entre la percepción subjetiva de la situación financiera del negocio y el ingreso del grupo familiar manifestado por los encuestados ( $p=0,0134)$, esto es, a mayor percepción subjetiva de la situación financiera del negocio, el porcentaje es cada vez mayor cuando crece el nivel de ingresos.

\section{Relación entre el estilo de vida / actitud hacia las entidades financieras y la percepción o valoración de la entidad}

A través de la variable 34 del cuestionario, desde el punto de vista cualitativo se ha tratado de medir la percepción que tienen los microempresarios de la confección textil con respecto a las características o atributos de su entidad financiera; con los resultados obtenidos se ha realizado una tabulación cruzada con los grupos del estilo de vida y actitud hacia las entidades financieras, mediante el algoritmo k-medias con análisis de componentes principales previo. Según el valor de $\mathrm{p}=0,4431$ correspondiente a un Ji cuadrado con 32 grados de libertad, se deduce que no existe dependencia entre las variables analizadas. 


\section{Conclusiones}

\section{De la hipótesis 1}

Sobre la base de la discusión de resultados, las técnicas de análisis multivariable, tales como el análisis de componentes principales y el análisis de conglomerados, han permitido segmentar el mercado microempresarial para el subsector de la confección textil, dividiendo en grupos claramente definidos y con características propias de acuerdo a la influencia de cada una de las variables. Según las pruebas lambda de Wilks, F de Snedecor y Ji cuadrado de Bartlett, se demuestra la pertinencia del análisis factorial aplicado a los diferentes constructos que explican el comportamiento de los microempresarios. De igual manera, la prueba F de Snedecor aplicada en el análisis de conglomerados (algoritmo de k-medias) prueba que los valores medios de cada grupo tienen diferencias significativas entre sí.

\section{De la hipótesis 2}

En la presente investigación no se pretende probar la relación causal entre los diferentes constructos planteados por Rodríguez Vargas, sino más bien explorar la dependencia entre los constructos pertenecientes a la zona de consumo y a la zona de endeudamiento. En la zona de "endeudamiento", según el análisis realizado referente a la relación entre la percepción subjetiva de la situación financiera y el constructo "factor personal" medido a través de la actitud al endeudamiento, mediante el algoritmo k-medias con análisis de componentes principales previo, se concluye que a un nivel de significancia del 6\%, se evidencia una dependencia entre estos constructos; no así, cuando la segmentación de la actitud al endeudamiento se realiza sin previo análisis de componentes principales.

Del mismo modo, con un nivel de significación del 1\% se concluye que existe una dependencia entre la percepción subjetiva de la situación financiera y el bienestar subjetivo de la conducta económica, medida a través de los hábitos y conductas de consumo caracterizado mediante el algoritmo k-medias, previo análisis de componentes principales; mas no cuando se compara 
con los grupos obtenidos sin previo análisis de componentes principales. Sin embargo, a un nivel de significación del 5\% se evidencia la dependencia.

En referencia a la relación entre el ingreso económico y la conducta económica, se demuestra que a un nivel de significancia del 5\% existe una dependencia entre los constructos, siempre y cuando se tenga un previo análisis de componentes principales en la conformación de los segmentos.

Del análisis realizado entre la relación de la percepción subjetiva de la situación financiera y el ingreso económico, medido a través del ingreso del grupo familiar, se concluye que a un nivel del $5 \%$ existe una dependencia.

En vista de que el estado de endeudamiento no fue ponderado en relación con las diferentes formas de endeudamiento, no fue posible establecer una correspondencia con el ingreso económico y el ambiente percibido de la situación financiera.

Por lo expuesto en los párrafos anteriores, es posible concluir que cinco de los seis constructos del modelo de Rodríguez Vargas muestran niveles de dependencia; por lo tanto, la aplicación al mercado microfinanciero del sector microempresarial de la industria de la confección textil es factible.

\section{De la hipótesis 3}

Según los resultados obtenidos, se puede evidenciar que no existe una relación de dependencia entre estilo de vida y actitud hacia las entidades financieras y la percepción o valoración que dan los microempresarios a los atributos de su entidad financiera; más bien, a un nivel de significación de $5 \%$ existe una independencia entre estas variables, que también se interpreta como que no existe una diferencia significativa entre los porcentajes de los atributos de la entidad financiera en los cinco grupos o segmentos de estilo de vida financiero. 


\section{Referencias}

Agell, Pieró, y José Antonio Segarra. 1997. Investigación de mercados. Barcelona: Estudios y Ediciones IESE.

Berger, Marguerite, Lara Goldmark y Tomás Miller-Sanabria. 2007. El boom de las microfinanzas: el modelo latinoamericano visto desde adentro. Nueva York: BID.

De Chocano, Juan. 2003. "Fundamentos psicológicos del comportamiento económico". Documento de trabajo, Colegio Oficial de Psicólogos de Madrid, Madrid. Consulta: octubre de 2015. 〈http://www.copmadrid.org/webcopm/publicaciones/trabajo/1990/vol2/arti3.htm».

Denegri, Marianela. 2006. Introducción a la psicología económica. Bogotá: PSICOM.

Denegri, Marianela, Mireya Palavecinos y María Ripoll. 1998. Consumir para vivir y no vivir para consumir. Temuco: Universidad de la Frontera.

Magill, John, y Richard Meyer. 2005. Microempresas y microfinanzas en el Ecuador: resultado del estudio de línea de base de 2004. Quito: SALTO / USAID.

Malhotra, Naresh K. 2004. Investigación de mercados: un enfoque aplicado. Ciudad de México: Pearson / Prentice Hall.

McCarthy, Jerome, y William Jr. Perreault. 2001. Marketing: un enfoque global. Ciudad de México: McGraw-Hill / Interamericana.

Rodríguez Vargas, Juan. 2006. "Validación del modelo psicoeconómico del consumidor". Pensamiento y Gestión, No. 20: 1-54.

Santesmases Mestre, Miguel. 2009. Diseño y análisis de encuestas en investigación social y de mercados. Madrid: Pirámide.

Schiffman, Leon, y Leslie Lazar Kanuk. 2005. Comportamiento del consumidor. Ciudad de México: Pearson.

Van Raaij, Fred. 1981. "Economic Psychology”. Journal of Economic Psychology 1, No. 1: $1-24$. 